\title{
Realization and application of 3D simulation system
}

\author{
Hongyuan Guo ${ }^{1, a}$ Yueguang Li ${ }^{2, b}$ \\ ${ }^{1}$ Gansu Normal University for Nationalities, Hezuo, gansu, 747000, \\ China \\ ${ }^{2}$ Gansu Normal University for Nationalities, Hezuo, gansu, 747000, \\ China \\ ${ }^{a}$ Yueguangli7@163.com, ${ }^{b}$ Yueguangli7@sohu.com
}

\begin{abstract}
:
Domestic and foreign technology, three dimensional simulation technology is a hot issue in recent years, its development change is rapidly. With the development of simulation technology, its application in the field of sports is more and more widely. This paper analyzes the application of simulation technology, builds simulation system and puts forward the method of updating simulation system, and uses the examples to introduce the 3D simulation system
\end{abstract}

Keywords: 3D simulation system; application; realization

\section{Introduction}

The aim of athletics is higher, faster and stronger, except the athletes hard training, scientific training method is an important and effective way to improve sports performance. Long term since, our country competitive sports training technical level is relatively low, it has been used the training and teaching methods based on experience, coaches with the naked eye and experience to guidance the athlete's skill, the athletes can only repeat practice to master the technical essentials, which seriously influence the further to rapidly improve the level of competitive sports in China ${ }^{[1]}$. Competitive sports teaching and training process, players need to have a large number of observation, imitation, feedback and correction of sensory signals, especially audiovisual sensations, characteristic of competitive sports technology of 3D simulation system has well make up for these 
deficiencies. Using this system the athletes can understand and master the details and key points of the top players of technical movements.

\section{Constitute the 3D simulation system}

\subsection{Virtual athletes}

This study refers to "the three dimensional digital virtual human" and "digital human" is not the same concept. The object of study of digital human body is a living, it is a dynamic human body system based on the multiple time and space, it is a technology system of human body real-time observation, network and computer information processing. The study object of "digitized virtual human" is dead, cut the corpse into thousands of slices with a knife and take pictures, carry on the integration in the computer, reconstruct 3D structure of human ${ }^{[2]}$. This is the difference between "digital human" and "digitized virtual human", it is only a link in the digital virtual technology. Study on "digitized virtual human" includes three research level of virtual visible human, virtual physical human and biological. The study purpose of digitized virtual human is the understanding of the situation of human body system in a certain time and space scales, its basic point is the living. he human body is composed of 100 trillion cells, only the nervous system has about 100000000000 neurons, the conflict and harmony of body and the external environment, these extremely complex changes of human body system is digitized virtual human can not be achieved, so the digital human of research in motion virtual reality is digital human based on living.

\subsection{The motion capture of technical information}

Motion capture technology is a new technology of using motion capture system of the athlete's skill and tactics or moving object three-dimensional movement locus of real-time capture and digital analysis. Motion capture system is a kind of high technology equipment of using for accurate measurement of moving object in the $3 \mathrm{D}$ space motion condition, the principle is based on computer graphics, capture device through the arrangement of a 
plurality of video in space, the movement condition of moving object (tracker) in image form recorded and processed by computer, get the space coordinate $(\mathrm{X}, \mathrm{Y}, \mathrm{Z})$ of different time and units of measurement the different objects ${ }^{[3]}$.

As shown in Figure 1, the motion capture equipment generally consists of sensors, signal capture equipment, data transmission equipment and data processing equipment. The sensor is tracking device of fixing on the specific parts of the moving object, provide the position information of moving to a system, the number of the tracker depends on the careful degree of motion capture; signal capture equipment is a hardware device of responsible for the acquisition and recognition analog signal of motion trajectory of sensors, the optical system is the high resolution infrared camera; data transmission equipment converts the analog signal into digital signal and the fast and accurate transmission to the computer system for processing; data processing equipment is composed of two parts: computer hardware equipment and data processing software, it is used to correct the data. The research uses Pose software to establish 3D human model, the establishment of bones and setting the function of joint, bone and model of human body skin and binding, a set of characters, make the court virtual space and environment, excellent athletes action simulation technology, nonlinear editing, rendering and output $3 \mathrm{D}$ action animation with the help of Maya software. As shown in figure $2^{[4]}$.

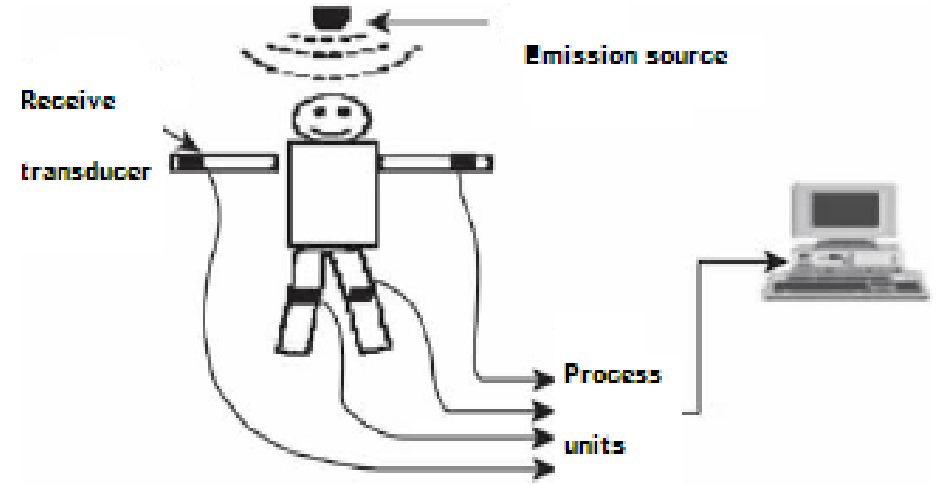

Fig. 1 Motion technology capture system 


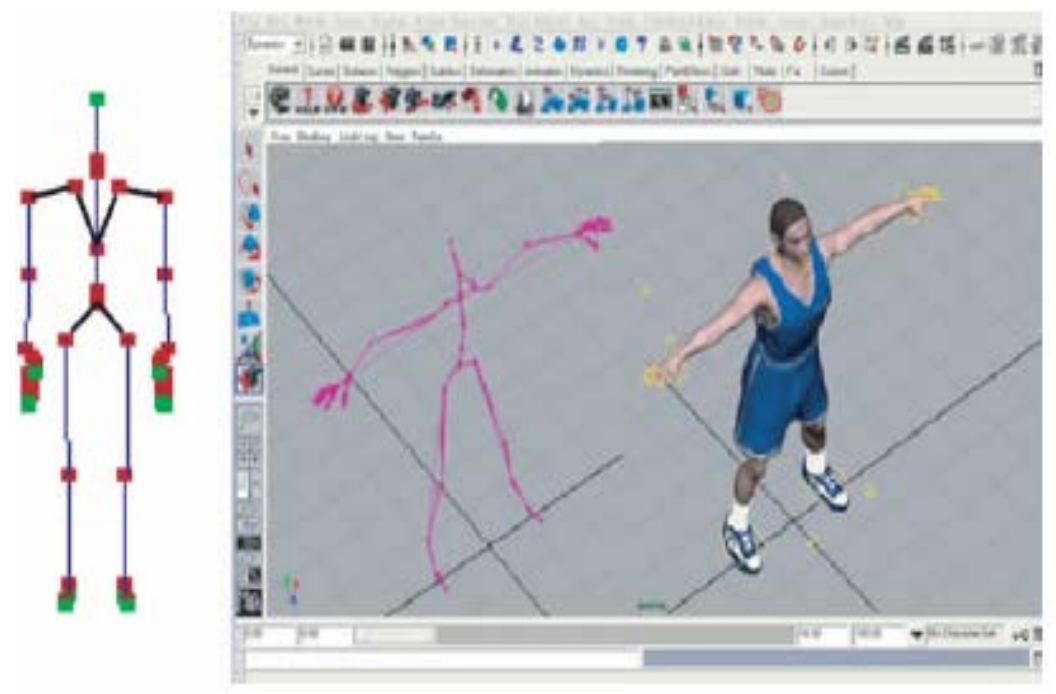

Fig. 2 Player character set

Motion capture technology can capture the action of basketball player, easy to carry on quantitative analysis, combined with human physiology, physics, improve the study methods, make physical training to get rid of the state of purely rely on the experience, enter the scientific, digital age. Capture the poor motion performance of athletes down, carries on the contrast analysis with excellent athletes' action, to help their training, providing accurate quantitative basis and training index of quantitative for scientific training. With the help of motion capture technology and computer image processing technology to greatly improve the visual technical training, and help to do quantitative analysis of technical action, display the results of the analysis, including the displacement, velocity, force (see Figure 3). On this basis, deeply analysis the "ideal" action and the athletes technical action and put forward opinions on the guiding action improvement of athletes ${ }^{[5]}$.

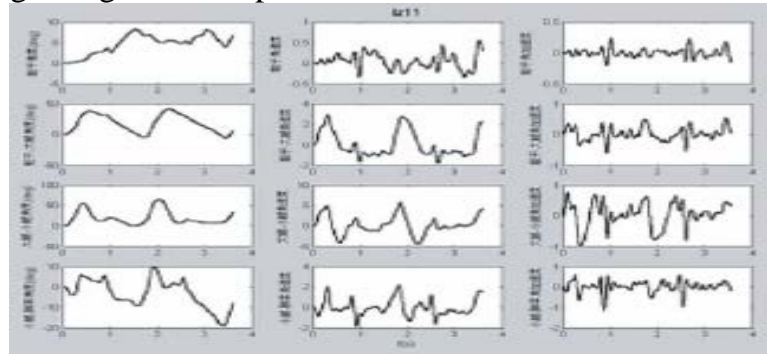

Fig. 3 Mechanical data of motion capture technology 


\subsection{Three dimensional simulation of movement of basketball technique}

Motion capture to obtain the instance database of a large number of technology actions, virtual human through Computer Research Institute of Chinese Academy of sciences research synthesis software package VHSDK5.0, show every example action in 3D visualization, and use the way of interaction design and modify the technical movement, and then validated Newton Euler model obtained from the rationality of the new movement, thus obtained personalized "standard" technical movement.

In order to facilitate completion of visual interactive action design, assume for human motion motion (t), the need to modify the attitude of posture $(\mathrm{t})$ to obtain the pose new posture $(\mathrm{t})$. In size of the $\mathrm{x}^{*} \mathrm{y}$ window, through the mouse and drag to select the corresponding rigid body for users. Drag the mouse hypothesis in the process of change in the $\mathrm{x}$ and $\mathrm{y}$ directions were $\Delta x$ and $\Delta y$ respectively, based on Euler's theorem assumes that the ZYX can be used to express the Euler angle $<\alpha \quad \beta \quad \gamma>$ this rotation, have the following relationship:

$$
\begin{aligned}
& \sin \alpha=\frac{\alpha \Delta x}{X}=\frac{(1-\alpha) \Delta y}{Y} \quad, \quad \sin \beta=\frac{(b \Delta x)}{X}=\frac{(1-\beta) \Delta y}{Y}, \\
& \sin \gamma=\frac{c \Delta x}{X}=\frac{(1-c) \Delta y}{Y}
\end{aligned}
$$

The a, b, c as the impact factor, says $\Delta \mathrm{x}$ and $\Delta \mathrm{y}$ respectively, the Euler angle $<\alpha \beta \quad \gamma \quad>$ in $\mathrm{x}, \mathrm{y}, \mathrm{z}$ influence degree of the three directions of the solution:

$$
\begin{aligned}
& \alpha=\arcsin \frac{a \Delta x+(1-a) \Delta y}{X+Y} \quad, \quad \beta=\arcsin \frac{b \Delta x+(1-b) \Delta y}{X+Y}, \\
& \gamma=\arcsin \frac{c \Delta x+(1-c) \Delta y}{X+Y}
\end{aligned}
$$

Complete the new attitude Posture (t) setting.

Adopt the new displacement mapping technology to obtain the movement of motion (t). Calculate the offset of $t$ moment: 
$d(t)=$ posture $^{\prime}(t)-$ posture $(t) \leq p_{0}^{\prime}(t)-p_{0}^{\prime}(t)$

Calculation the offset of other time as shown in Figure 4, obtained with motion $(t)$ corresponding to the motion offset.

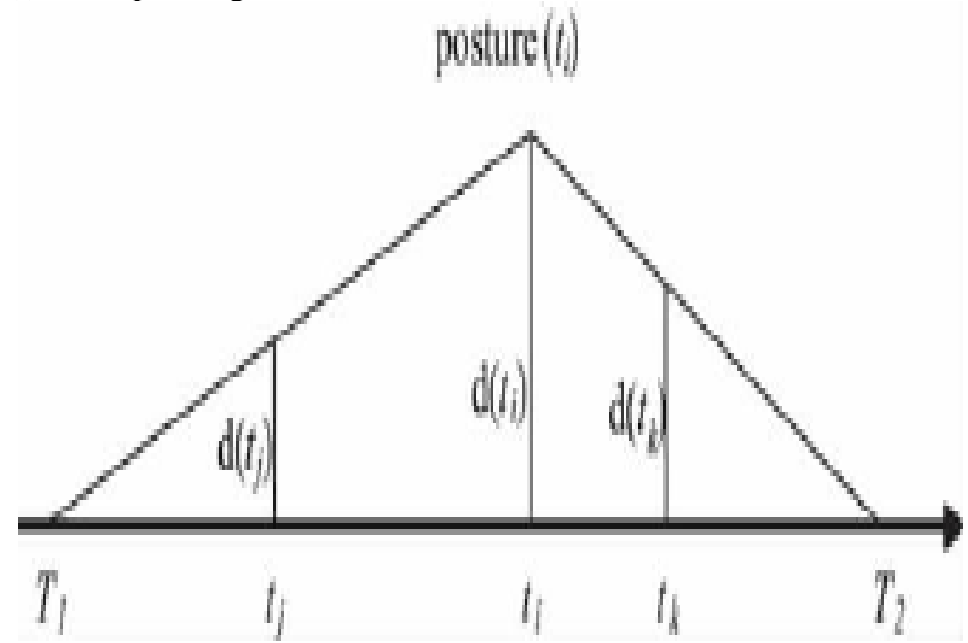

Fig. 4 Tectonic offset

According to the characteristics of athletes of the posture parameters to budget inertial parameters of human body, determine the moment of inertia and other personalized parameters in Newton Euler model need, get the equations of motion, according to the equation to verify the rationality of the new motion of motion (t) ${ }^{[6]}$.

\section{The design of the database}

Although Sohu and Sina have been established competitive sports database, but the database is to provide popular information as the goal, not to provide a team or a player as the goal of the search engine, and can't provide the datas of video clips related technical and tactical analysis. About competitive sports technical and tactical, it has not found related and more mature professional competitive sports technology of database design report. For effective management of the mass competitive sports technical data, choose the 
construction of competitive sports technology database is the only choice. Competitive sports technology database system includes the following parts: database, hardware, software and human. Database administrators are responsible for the definition, establishment and maintenance of database; main duties of database application programmers is that use the system to develop the database, in order to realize the database establishment of information, information retrieval, delete or change existing information, or produce various forms of report; Database Operator don't write process program with the language or tool, the direct use the command or query language for direct access and operation database operation. Competitive sports technology of 3D simulation database should integrate classic technology of many domestic and foreign high level basketball players, using query and search commands can be achieve the plane preview and three-dimensional output demo of different basketball player movement, dribble, pass, shoot, rebound, many personal defense technology.

\section{Conclusions}

Research on 3D simulation technology of competitive sports teaching and training in modern competitive sports skills enormous effect, it can do quantitative analysis on the technical action and graphically display the results of the analysis, on the basis, do deep analysis on the "ideal" action and the athletes technical action, the construction of virtual environment of athletes in training and competition, make up for the reason of weather, venue, equipment, funds or athletes injury as a result in negative effects.

\section{References}

[1] Chen Jian. Research on the basketball tactics teaching software of modern computer information technology development[J]. Journal of the University of Hangzhou Normal University (natural science edition), 2006 (5): 38-40. 
[2] Sun Yongchao. Research on scheduling method of virtual athletes of trampoline movement in simulation [R]. ADO the first intelligent and Digital Entertainment Conference, 2003.

[3] Chen Jian. The application of virtual reality technology in the sports technique simulation[J]. Sports Science, 2006 (9): 97-101.

[4] Feng Lizheng,Chen Jian. Application of motion capture technology in sports[J]. Journal of the University of Hangzhou Normal University, 2005 (3): 21-23.

[5] Chen Jian. Motion capture technique and its application in the diagnosis of Sports Technology[J]. Journal of Shanghai Institute of Physical Education, 2006 (4): 46-49.

[6] Chen Jian. Research and development of multimedia database of basketball technique and tactics [J]. Journal of Shanghai Institute of Physical Education, 2007 (4): 29-31. 